\title{
Burst detection in turbulent channel flows based on large eddy simulation databases
}

\author{
ZHANG Qiang, ZHOU Jifu \& LI Jiachun \\ Institute of Mechanics, Chinese Academy of Sciences, Beijing, 100080 \\ Correspondence should be addressed to Zhang Qiang (email: zhangtsiang@21cn.com) \\ Received April 24, 2005
}

\begin{abstract}
Reliable turbulent channel flow databases at several Reynolds numbers have been established by large eddy simulation (LES), with two of them validated by comparing with typical direct numerical simulation (DNS) results. Furthermore, the statistics, such as velocity profile, turbulent intensities and shear stress, were obtained as well as the temporal and spatial structure of turbulent bursts. Based on the LES databases available, the conditional sampling methods are used to detect the structures of burst events. A method to deterimine the grouping parameter from the probability distribution function (pdf) curve of the time separation between ejection events is proposed to avoid the errors in detected results. And thus, the dependence of average burst period on thresholds is considerably weakened. Meanwhile, the average burst-tobed area ratios are detected. It is found that the Reynolds number exhibits little effect on the burst period and burst-to-bed area ratio.
\end{abstract}

Keywords: turbulence, burst period, burst-to-bed area ratio, LES.

DOI: $10.1360 / 142004-4$

As one of the important coherent structures in the near-wall region, turbulent burst is responsible for the production and transport of major turbulent kinetic energy and Reynolds stress ${ }^{[1]}$. Nearly half of turbulent kinetic energy or Reynolds stress is produced in the near-wall region, and $80 \%$ flows in outer region only contribute $20 \%$ of them. Both ejection and sweeping events contribute $60-70 \%$ of the turbulent shear stress respectively $^{[2]}$. Recently, turbulent burst process has been found to be closely related to scalar transport. Taking sediment transport for example, Gyr \& Schmid ${ }^{[3]}$ showed that the movement of sediment near bed is mainly affected by the lift and breakdown of low-speed streaks in the near-wall region. Nino et al. ${ }^{[4]}$ found that sediment motion is closely associated with the turbulent bursts. In the region near a smooth wall, the sediment particles, especially the finer ones, accumulate around the low-speed streaks and are entrained into flows. The angles to the wall at which the particles are picked up are about $10^{\circ}-20^{\circ}$, very close to the incline angle of the shear layer between high and low speed fluids in the near-wall region, namely about $14^{\circ}$. In view of sediment motion in 
coastal areas, more fundamental understanding of turbulent bursting in unsteady flows is necessary.

Because of aforementioned reasons, numerous techniques were devised to detect the characteristics of the burst structure, among which $u v$ quadrant $2\left((u v)_{2}\right)$, VITA and mu-level are the most common methods to detect the average burst period. Generally, there is at least one adjustable parameter in each of them. In turn, this would cause the discrepancy between the detected results. Therefore, some modifications to these techniques, or some more objective methods such as wavelet transform, were adopted ${ }^{[5]}$. In addition, Bogard and Tiederman ${ }^{[6]}$ found that the burst originated from one streak might contain one or more ejection events. Hence, they introduced a parameter, called grouping parameter, to group and separate the ejection events. Two consecutive ejection events would belong to the same burst event if the time separation between them is less than the grouping parameter. Otherwise, they would belong to different burst events respectively. Luchik \& Tiederman ${ }^{[7]}$ carried out similar experimental studies. However, the way to determine the grouping parameter needs further exploration.

On the other hand, burst was mainly studied by experiments in the history. There are very few studies on burst based on the numerical simulations. In experiments, only time series at very limited points could be acquired. On the contrary, almost full, spatial and temporal flow properties, including the statistics and the spatial and temporal variations of the burst structures could be presented in a numerical simulation. The Reynolds-Averaged Navier-Stokes Equations modeling (RANS) among them is obviously improper for detecting the burst because the fluctuations are averaged. Only the methods preserving enough fluctuations could be used to detect the burst events, such as LES or DNS, which make it possible to capture the streak structure and the burst events in the near-wall flows. Even so, the simulation has to offer enough resolution in the near-wall region. Based on their DNS results of the flat-plate boundary layer at low Reynolds number, Kim \& Spalart ${ }^{[8]}$ studied the spatial frequency of occurrence of the bursting process, and estimated the average burst period through the propagation speed of the structure by the Taylor theory. In their Reynolds number range, the spatial frequency of occurrence of the burst scales with the inner variables, and so does the temporal one, which agrees well with Luchik \& Tiederman $^{[7]}$. Li et al. ${ }^{[8]}$ drew a similar conclusion by analyzing their DNS results based on the wavelet transform.

In this paper, we use LES with the grids refined in the near-wall region to reach enough resolution, which guarantees that the important coherent structure-low-speed streak-is simulated accurately. We compare the results with typical DNS data for validation. In addition, computation convergence or grid independence is tested to achieve consistent results. Channel flows at several Reynolds numbers are simulated, and then detailed databases are established. We have worked out a method to determine the grouping parameter from the pdf curve of the time separations between ejection events. Determining the grouping parameter in this manner, we obtain the average burst 
periods at different Reynolds numbers, as well as the average burst-to-bed area ratios.

\section{LES databases of channel flows}

The databases of channel flows are established by LES in which turbulence is decomposed into geometry-dependent large-scale part $\bar{f}$ and relatively universal small-scale part $f^{\prime}$ by filtering process, i.e. $f=\bar{f}+f^{\prime}$. The large-scale motions are solved numerically while the small-scale ones are modeled with so-called sub-grid scale (SGS) models. Furthermore, $\langle\bar{f}\rangle$ stands for horizontal as well as temporal averaging and $\bar{f}^{\prime}$ for $\bar{f}-\langle\bar{f}\rangle$.

For the incompressible fluid, the governing equations for the large-scale motions are

$$
\begin{gathered}
\frac{\partial \bar{u}_{i}}{\partial x_{i}}=0 \\
\frac{\partial \bar{u}_{i}}{\partial t}+\frac{\partial \bar{u}_{i} \bar{u}_{j}}{\partial x_{j}}=-\frac{1}{\rho} \frac{\partial \bar{p}}{\partial x_{i}}-\frac{\partial Q_{i j}}{\partial x_{i}}+v \frac{\partial^{2} \bar{u}_{i}}{\partial x_{j} \partial x_{j}}
\end{gathered}
$$

where $Q_{i j}=\overline{u_{i} u_{j}}-\bar{u}_{i} \bar{u}_{j}$ is the SGS stress, which needs modeling. The Smagorinsky model is adopted here

$$
\tau_{i j}=Q_{i j}-\frac{\delta_{i j}}{3} Q_{k k}=-2 v_{T} \bar{S}_{i j}
$$

where

$$
S_{i j}=\frac{1}{2}\left(\frac{\partial \bar{u}_{i}}{\partial x_{j}}+\frac{\partial \bar{u}_{j}}{\partial x_{i}}\right), \quad v_{T}=\left(C_{s} \Delta l_{s}\right)^{2}|\bar{S}|,|\bar{S}|=\sqrt{S_{i j} S_{i j}},
$$

in which $\Delta$ is the filter width and $C_{s}$ is a constant set to be 0.1 . Because walls inhibit the sizes of the energy-containing eddies, Van Driest damping function, $l_{s}=1-\exp \left(-y^{+} / 25\right)$, is used to account for such effects.

Also, we introduce the following variables

$$
\bar{\omega}_{i j}=\varepsilon_{i j k} \frac{\partial \bar{u}_{i}}{\partial x_{j}}, \quad Q_{i j}=\overline{u_{i} u_{j}}-\bar{u}_{i} \bar{u}_{j}, \quad P=\frac{\bar{p}}{\rho}+\frac{1}{2} \bar{u}_{i} \bar{u}_{j}+\frac{1}{3} Q_{k k} .
$$

The friction velocity and channel half-width are the characteristic scales for non- dimensionalization

$$
\frac{\partial \bar{u}_{i}}{\partial t}-\varepsilon_{i j k} \bar{u}_{i} \bar{\omega}_{k}=-\frac{\partial P}{\partial x_{i}}-\frac{\partial \tau_{i j}}{\partial x_{i}}+\frac{1}{R e_{\tau}} \frac{\partial^{2} \bar{u}_{i}}{\partial x_{j} \partial x_{j}}+\delta_{1 i},
$$




$$
\frac{\partial \bar{u}_{i}}{\partial x_{i}}=0
$$

where $\delta_{1 i}$ is the streamwise pressure gradient, $R e_{\tau}=u_{\tau} \delta / v$ is the Reynolds number.

Pseudo-spectral method (de-aliasing by the rule 3/2) is used in horizontal directions with periodical boundary conditions. A finite difference scheme is used in the normal direction on a non-uniform staggered mesh. A Poisson's equation is solved for $P$. The Adams-Bashforth scheme is utilized for time evolution.

The grids are refined in the near-wall region. In the lower half channel $(0<y<\delta$, or 0 $<y<1$ if non-dimensionalized by $\delta$ ), the stretching formula is written as

$$
y_{j}=\frac{e^{\alpha \xi_{j}}-1}{e^{\alpha}-1}, \quad \xi_{j}=\frac{j}{N_{y} / 2},
$$

where $N_{y}$ is the number of grids in the wall-normal direction. The grids of the other half are given by the symmetry. $a$ is the stretching parameter to adjust the density of grids. The higher the value of $a$, the finer the grids near the walls, and the closer to the wall the first grid-layer. To simulate the near-wall flows sufficiently, Zang ${ }^{[10]}$ proposed the basic requirements for grid resolution, i.e. the streamwise spacing in wall units $\Delta x^{+}<80$, the spanwise spacing $\Delta z^{+}<30$, and in the wall-normal direction, there are at least 3 grid points in the region of $y^{+}<10$. Furthermore, Piomelli ${ }^{[11]}$ suggested that in order for the near-wall structure to be sufficiently resolved in LES, the first grid point in the normal direction should be located at $y^{+} \leqslant 1.0$, and $\Delta x^{+} \quad 50-150, \Delta z^{+} \quad 15-40$. It is convinced that there is little distinction between LES and DNS if the near-wall flows are well simulated.

To begin with, we have simulated the channel flows at $R e_{\hat{o}}=180$ and 395 and compared with typical DNS results as validations. Then, the simulation of the channel flow at $R e_{\hat{o}}=300$ is carried out to examine Reynolds number effects. The computational parameters are shown in Table 1 . The grid systems meet the requirements suggested by

\begin{tabular}{|c|c|c|c|}
\hline $\operatorname{Re}_{\hat{o}}$ & 180 & 300 & 395 \\
\hline Domain & $2.5 \pi \delta \times 2 \delta \times 1.5 \pi \delta$ & $2.5 \pi \delta \times 2 \delta \times 3 \pi \delta / 4$ & $2.5 \pi \delta \times 2 \delta \times 2 \pi \delta / 3$ \\
\hline$N_{x} \times N_{y} \times N_{z}$ & $64 \times 64 \times 64$ & $64 \times 64 \times 64$ & $64 \times 64 \times 64$ \\
\hline$A$ & 2.0 & 2.5 & 3.0 \\
\hline$\Delta x^{+}$(streamwise spacing, in wall units) & 22 & 37 & 48 \\
\hline$y_{1}^{+}$(the first grid layer, in wall units) & 0.9 & 1.0 & 1.0 \\
\hline$\Delta z^{+}$(spanwise spacing, in wall units) & 13 & 11 & 13 \\
\hline$\Delta t$ (time step, normalized by $\mathrm{u}_{\hat{o}}$ and $\left.\ddot{a}\right)$ & 0.001 & 0.001 & 0.001 \\
\hline$\Delta t^{+}=\Delta t \cdot \operatorname{Re}_{\hat{o}}($ time step, in wall units) & 0.18 & 0.3 & 0.395 \\
\hline$N_{T}$ (number of integral time steps) & 30000 & 30000 & 30000 \\
\hline$T$ (integral time, normalized by $\mathrm{u}_{\hat{o}}$ and ä) & 30 & 30 & 30 \\
\hline$T^{+}$(integral time, in wall units) & 5400 & 9000 & 11850 \\
\hline
\end{tabular}

Table 1 Computational parameters of LES of channel flows 
Piomelli ${ }^{[11]}$. Meanwhile, the time step is an analog to the sampling period in experiments. The time steps $\left(\Delta t^{+}\right)$are less than the time scale to be detected $\left({T_{B}}^{+}\right.$, see later). In the result of $\mathrm{Li}$ et al. ${ }^{[9]}, \Delta t^{+} \quad 0.11$, and in the experimental result of Jiang et al. ${ }^{[12]}, \Delta t^{+}$ 0.512 . The time steps in this paper are similar to these data, indicating that the time resolutions are adequate too.

The results of channel flow at $R e_{\tau}=180$ are shown in Figs. $1-4$. Fig. 1 presents the turbulent intensities. The agreement with the results by Kim et al. ${ }^{[2]}$ (indicated by

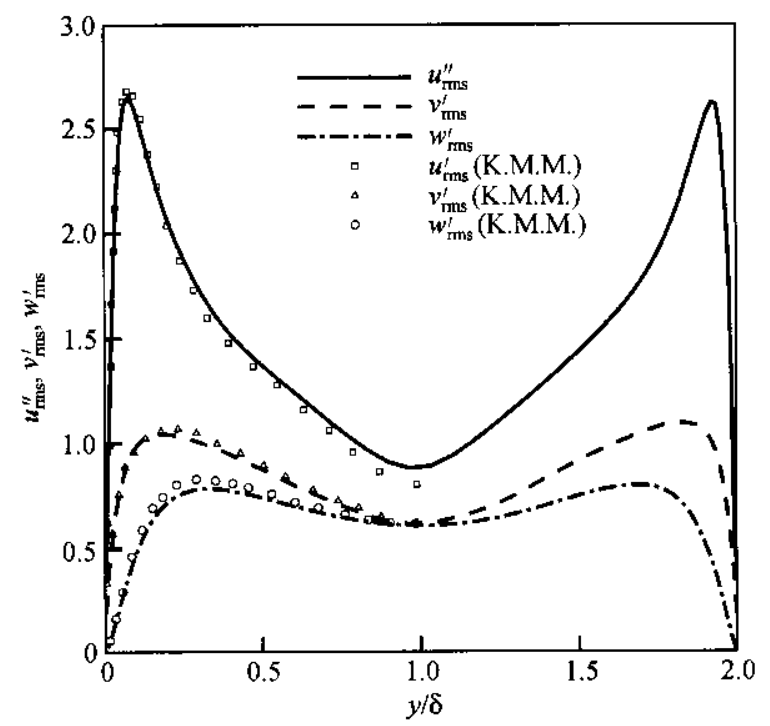

Fig. 1. Turbulent intensities, $R e_{\tau}=180$.

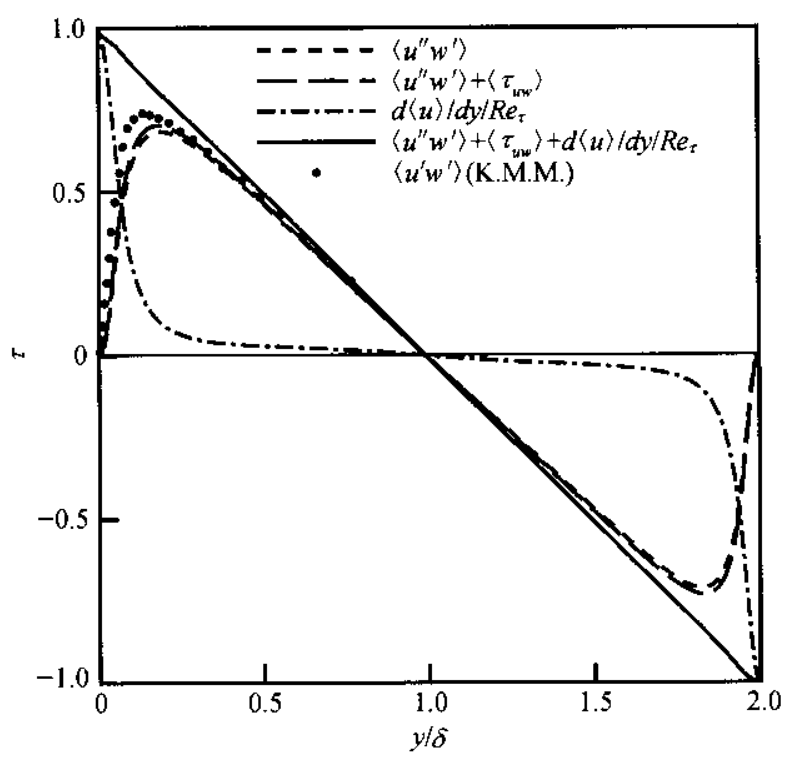

Fig. 2. Shear stresses, $R e_{\tau}=180$. 


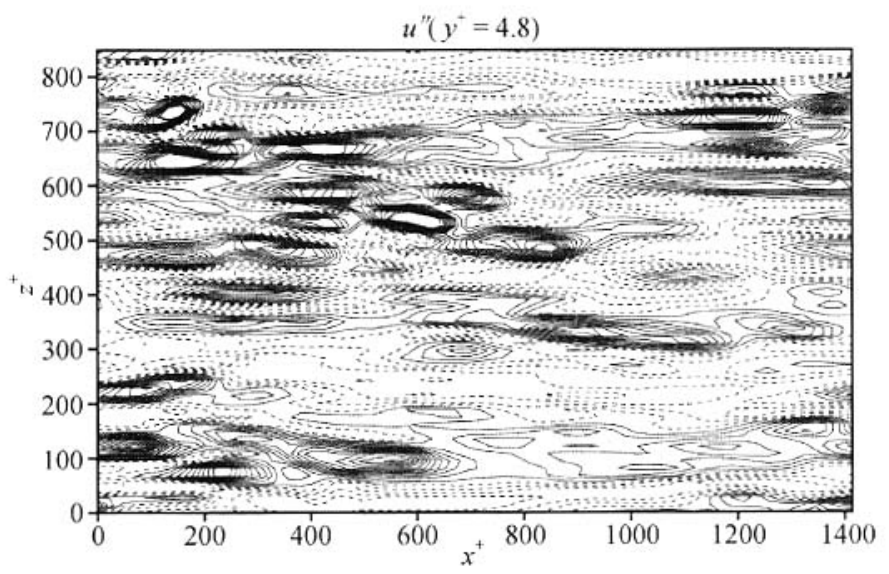

Fig. 3. Contours of streamwise fluctuations near the wall, $y^{+}=4.8, R e_{\tau}=180$.

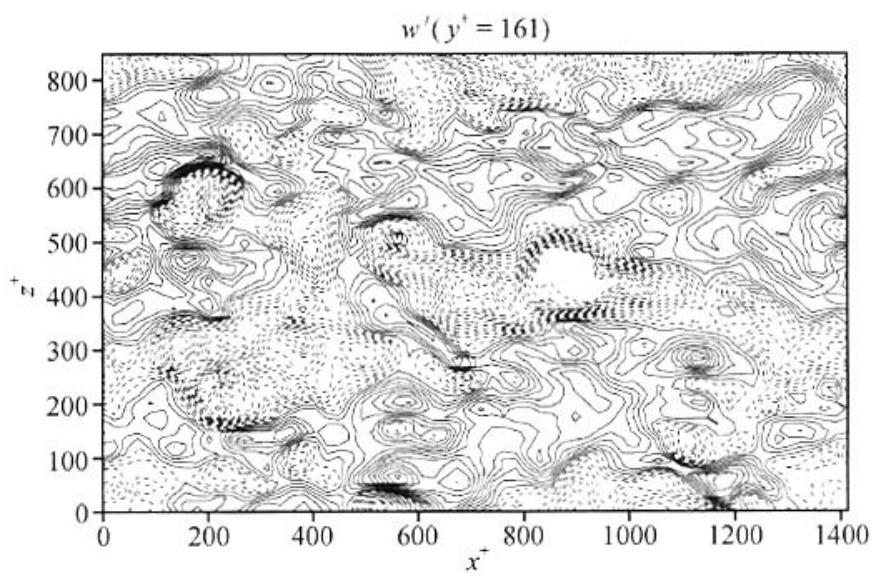

Fig. 4. Contours of streamwise fluctuations near the center-line, $y^{+}=161, R e_{\tau}=180$.

K.M.M.) is good. The symmetry of the distribution of turbulent intensities about the channel center-line proves that the integral time is long enough. The stress distributions are shown in Fig. 2. The solid line denotes the sum of large scale Reynolds stress $\left(-\left\langle\bar{u}^{\prime \prime} \bar{w}^{\prime}\right\rangle\right)$, SGS stress $\left(\tau_{u w}\right)$ and viscous stress $\left(d\langle u\rangle / d y / R e_{\hat{o}}\right) . \bar{u}^{\prime \prime}=\bar{u}-\langle\bar{u}\rangle$ denotes the streamwise large-scale fluctuations, $\bar{v}^{\prime}=\bar{v}$ and $\bar{w}^{\prime}=\bar{w}$ are the large-scale fluctuations in the spanwise and normal directions respectively. This profile shows that the average shear stress balances the streamwise mean pressure gradient. It can also be seen that the SGS stress only occupies small part of total stress (less than 20\%). In turn, the turbulent kinetic energy produced by SGS stress occupies small part of total kinetic energy too. Therefore, the effect of SGS motions on the large-scale motions is substantially small, and would not significantly affect large-scale structures, such as burst events. Figs. 3 and 4 present the contours of streamwise fluctuations near the wall and about the center-line, respectively. The streak structures can be identified clearly in the near-wall region and disappear in the channel center. The average spanwise spacing between low-speed 
streaks is approximately 120 in wall units, in agreement with the results of Kim et al. ${ }^{[2]}$ and Kline et al. ${ }^{[1]}$.

Similarly, the results of channel flow at $R e=395$ are shown in Figs. 5 and 6 . The data compared with are the DNS results by Moser et al. ${ }^{[13]}$, indicated by M.K.M. in the figures. By these comparisons, we have validated the current LES computation.

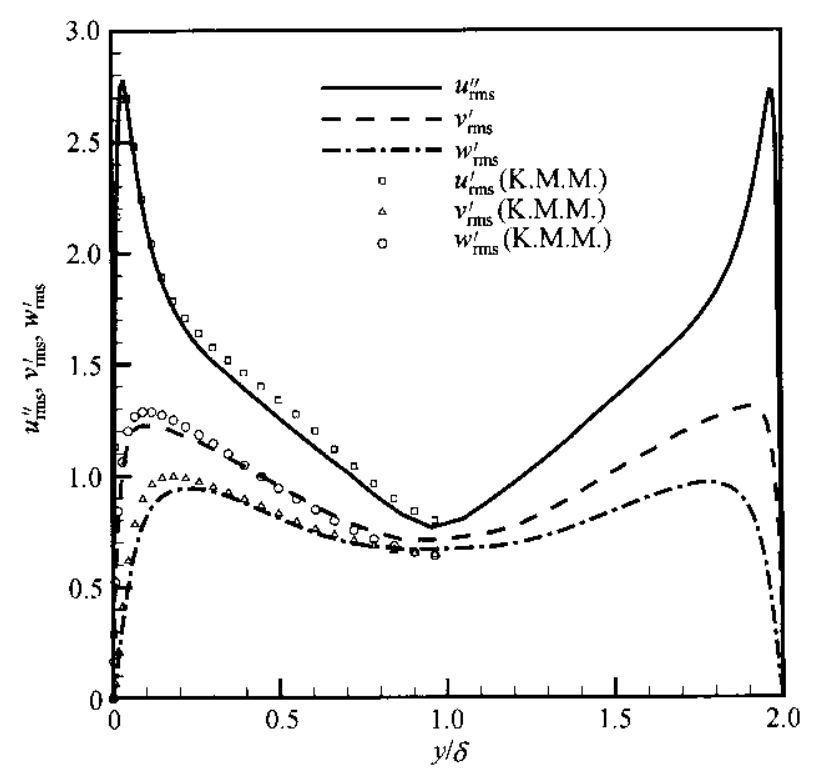

Fig. 5. Turbulent intensities, $R e_{\tau}=395$.

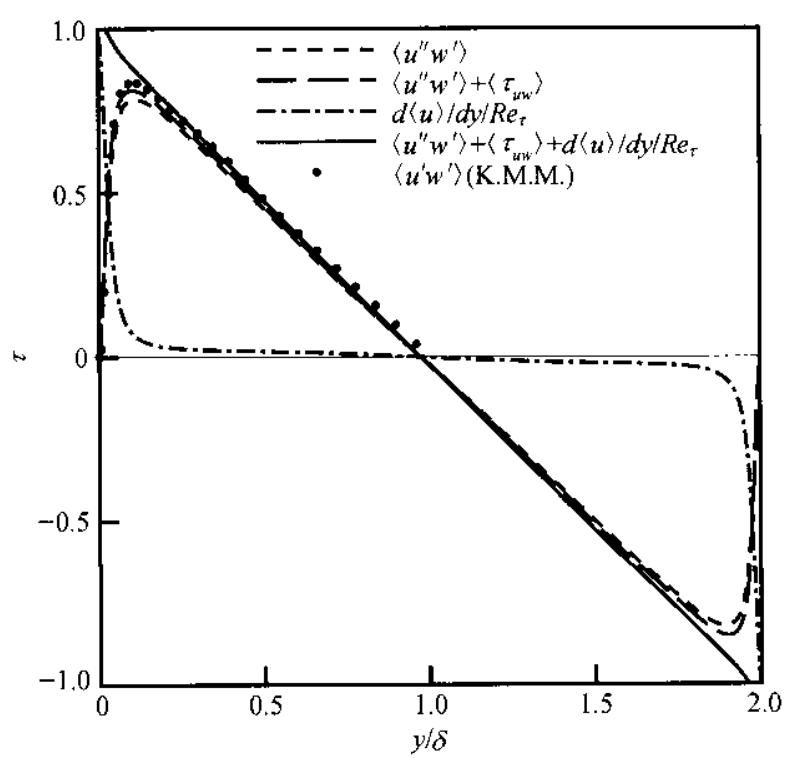

Fig. 6. Shear stresses, $R e_{\tau}=395$. 
In addition, computational convergence is tested for $R e_{\hat{o}}=180$. We refine the grids in both horizontal direction and in wall-normal direction by a factor of 2. Fig. 7 presents the comparison of stresses between the cases of $N_{x}=N_{z}=64$ and $N_{x}=N_{z}=128$. No significant changes are observed. It is seen that there is little change in stress. Only SGS part becomes smaller, indicating that the finer the grids, the smaller the effect of SGS motions on the large-scale ones. The comparison of turbulent intensities of the two cases is shown in Fig. 8, where the 5\% error band (denoted by "I") of the case of $N_{x}=N_{z}=64$ is also given. With grid refined, the change is smaller than $5 \%$.

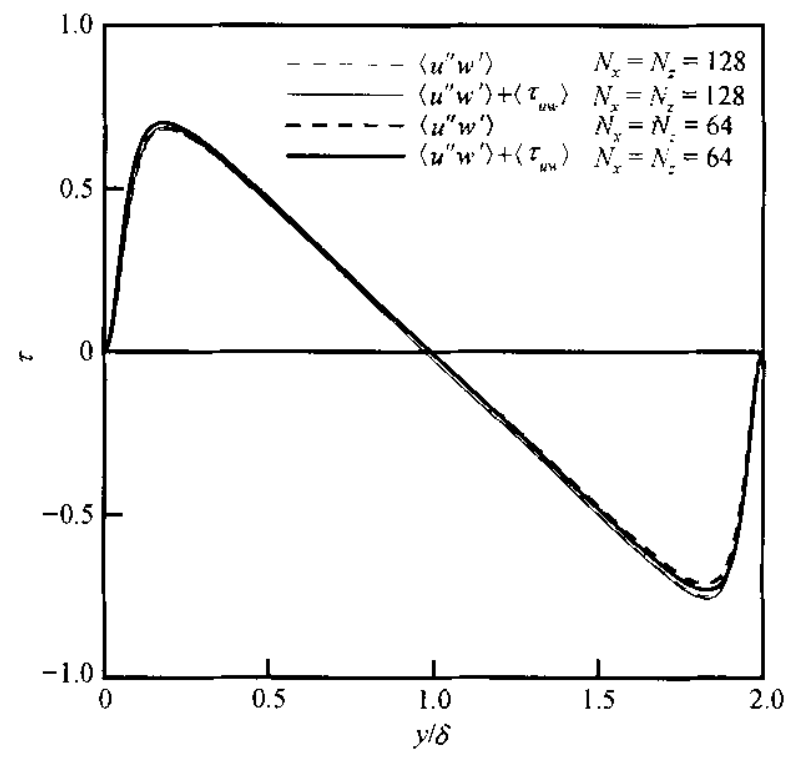

Fig. 7. Computational convergence for stress, $R e_{\tau}=180$.

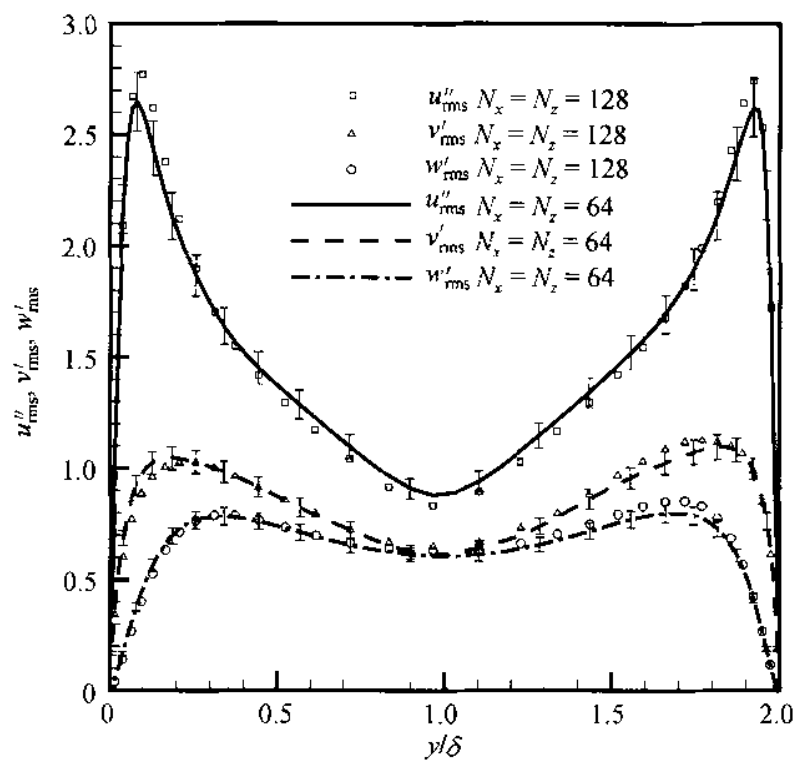

Fig. 8. Computational convergence for stress, $R e_{\tau}=180$. 


\section{Burst detection}

\subsection{Detection techniques}

Three commonly used techniques for burst detection are $u v$ quadrant $2\left((u v)_{2}\right)$, mu-level and VITA. Each of them has its own empirically determined threshold. Therefore, the detected results are more or less subjective.

Luchik \& Tiederman ${ }^{[7]}$ compared them in detail based on experimental data. They argued that, with grouping parameter, all these techniques provide reasonable results and in particular, $(u v)_{2}$ and mu-level bear wider threshold-independent region than VITA if long time series is available. Therefore, only $(u v)_{2}$ and mu-level are adopted here. The details of them are shown in Table 2 , where $\vec{u}^{\prime}, \vec{v}^{\prime}$ denote the large-scale fluctuations in the streamwise and normal directions respectively, and the subscript rms denotes the root-mean-square; $\left(\bar{u}^{\prime} \bar{v}^{\prime}\right)_{2}$ is the $\bar{u}^{\prime} \bar{v}^{\prime}$ in the quadrant 2 , and $L, H$ are thresholds.

Table 2 The details of $(u v)_{2}$ and mu-level techniques

\begin{tabular}{llc}
\hline & \multicolumn{1}{c}{ characteristics } & Sampling function \\
\hline$(u v)_{2}$ & $\begin{array}{l}\text { Detecting motions associated with an ejec- } \\
\text { tion event, i.e. } \bar{u}^{\prime}\left\langle 0, \bar{v}^{\prime}\right\rangle 0\end{array}$ & $D(t)= \begin{cases}1, & \left|\left(\bar{u}^{\prime} \bar{v}^{\prime}\right)_{2}\right|>H \bar{u}_{\mathrm{rms}}^{\prime} \\
0, & \text { otherwise }\end{cases}$ \\
mu-level & $\begin{array}{l}\text { Detecting deficits in the mean streamwise } \\
\text { velocity }\end{array}$ & $D(t)= \begin{cases}1, & \bar{u}^{\prime}<-L \bar{u}_{\mathrm{rms}}^{\prime} \\
0, \bar{u}^{\prime}>-0.25 L \bar{u}_{\mathrm{rms}}^{\prime}\end{cases}$ \\
\hline
\end{tabular}

\subsection{Average burst period}

Based on experimental data, Luchik \& Tiederman ${ }^{[7]}$ concluded that the average burst period scales with the inner variables, i.e.

$$
T_{B}^{+}=T_{B} \cdot u_{\tau}^{2} / v \approx 90
$$

where $u_{\tau}, v, T_{B}, T_{B}^{+}$are friction velocity, viscosity, burst period with dimension and non-dimensionalized burst period.

Scholars are divided on what variables (inner, outer or mixed) the burst period scales with. Blackwelder \& Haritonidis ${ }^{[14]}$, Luchik \& Tiederman $^{[7]}$ suggested that inner variables are proper ones. However, Alfredsson \& Johansson ${ }^{[15]}$ claimed that the bursts result from the strong interaction between the flows near the wall and the outer flows. Therefore, the burst period should scale with the mixed variables. Shah \& Antonia ${ }^{[16]}$ experimentally investigated the boundary layers and duct flows at a wide range of Reynolds number. According to Shah \& Antonia ${ }^{[16]}$, the burst period scales with inner variables $^{[7-9,14]}$ in the lower Reynolds number flows, whereas it scales with the mixed variables in the flows of higher Reynolds numbers. Limited by the computational capacity, the Reynolds numbers in this paper are fairly low. Hence, we tend to compare them with Luchik \& Tiederman's results ${ }^{[7]}$.

The variables in the current simulation are non-dimensionalized by $u_{\hat{o}}, \delta$ (friction 
velocity and channel half-width). When non-dimensionalized by inner variables, one gets $T_{B}^{+}=\operatorname{Re}_{i} T_{B N}$, where $T_{B N}$ is the burst period detected directly from the simulation data. Therefore according to eq. (9), $T_{B N} \quad 0.5$ for $R e_{\tau}=180, T_{B N} \quad 0.3, \operatorname{Re}_{\tau}=300$, and so on.

According to Bogard \& Tiederman ${ }^{[5]}$, a burst process originated from one low-speed streak contains one or more ejection events. They introduced a grouping parameter to group the consecutive ejection events belonging to the same burst process and separate those belonging to different burst processes. It is convinced that the time separations between ejection events belonging to different burst processes follow different probability distributions. In Fig. 9, Luchik \& Tiederman ${ }^{[7]}$ showed the idealized probability distribution of time separations between ejections. The left part of the curve in Fig. 9 was deemed as the pdf of the time separations between ejections from the same burst, and the right part as the pdf of those from different bursts. Ideally, there is a clear separation between two types of distribution, as $\tau_{\mathrm{E}}$ shown in the figure, which is chosen to be the grouping parameter. Actually, Luchik \& Tiederman ${ }^{[7]}$ set $\tau_{E}$ from the accumulation distribution curve of the time separations between ejections, as shown in Fig. 10. Luchik \&

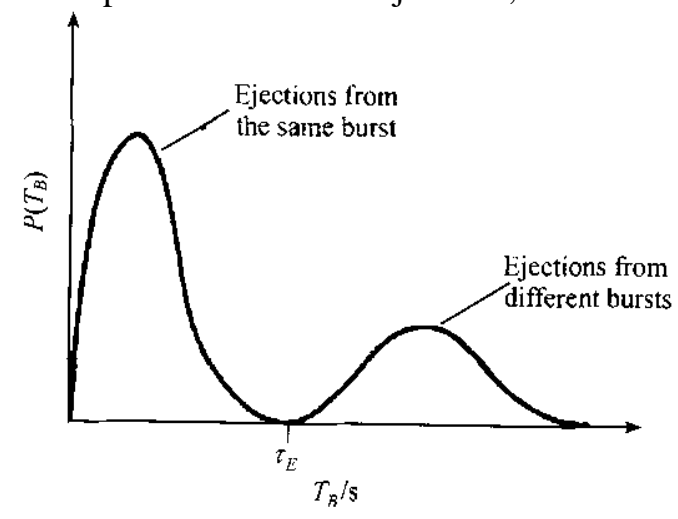

Fig. 9. Idealized pdf of time between ejections from Luchik \& Tiederman ${ }^{[7]}$.

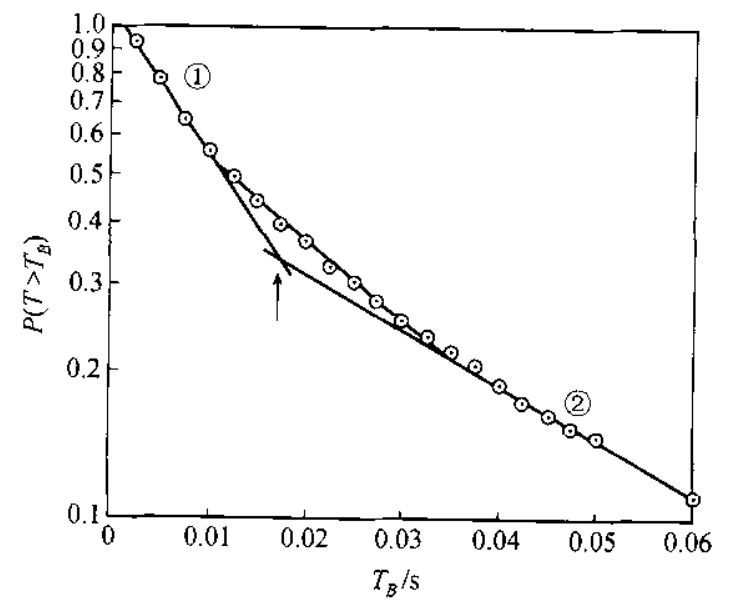

Fig. 10. Method to determine the grouping parameter by Luchik \& Tiederman ${ }^{[7]}$ for $(u v)_{2}$. 
Tiederman $^{[7]}$ found that two types of time separations follow the exponential distributions with different characteristic parameters. In Fig. 10, (1) denotes the cumulative distribution curve of the time separations between ejections from the same burst, and (2) denotes that from different bursts. In this kind of semi-log plot, two straight lines were fitted for (1) and (2), and the abscissa value of the meeting point was chosen by Luchik $\&$ Tiederman $^{[7]}$ as the grouping parameter. It is easy to group or separate ejections with the grouping parameter. If the time separation between two consecutive ejections, $T_{E}$ is larger than $\tau_{E}$, i.e. $T_{E}>\tau_{E}$, the two ejections belong to different bursts; otherwise, they belong to the same burst. However, it is not easy or somewhat arbitrary to fit the lines for (1) and (2) when data are scarce.

In this paper, we find the grouping parameter from the pdf curve. Firstly, a threshold is chosen in the common range for the two techniques to detect ejection events in turbulent signal series from the current LES database, and the time separations between ejections were calculated, as well as their pdf. Then, average from 5 points is carried out to smooth the distribution curve as shown in Fig. 9. The value of $\hat{o}_{E}$ can be determined easily this way.

With the grouping parameter introduced, the results of burst period are evidently improved. Fig. 11 shows the comparison between the results with and without grouping parameter for $(u v)_{2}$ method at $R e_{\tau}=180$ and $y^{+}=14.1$. Without the grouping parameter, all the ejections detected are regarded as bursts, which are more than actual bursts in number. Therefore, the average burst period is lower and dependent on the threshold to a great extent. For example, only when $H=1.4$, can reasonable period $\left(T_{B N}=0.5\right)$ be obtained. However, with the grouping parameter, the average burst period augments. The threshold-independent region can be found in the curve, as shown in Fig. 11, when $0<H$ $<1.0, T_{B N} \approx 0.5$.

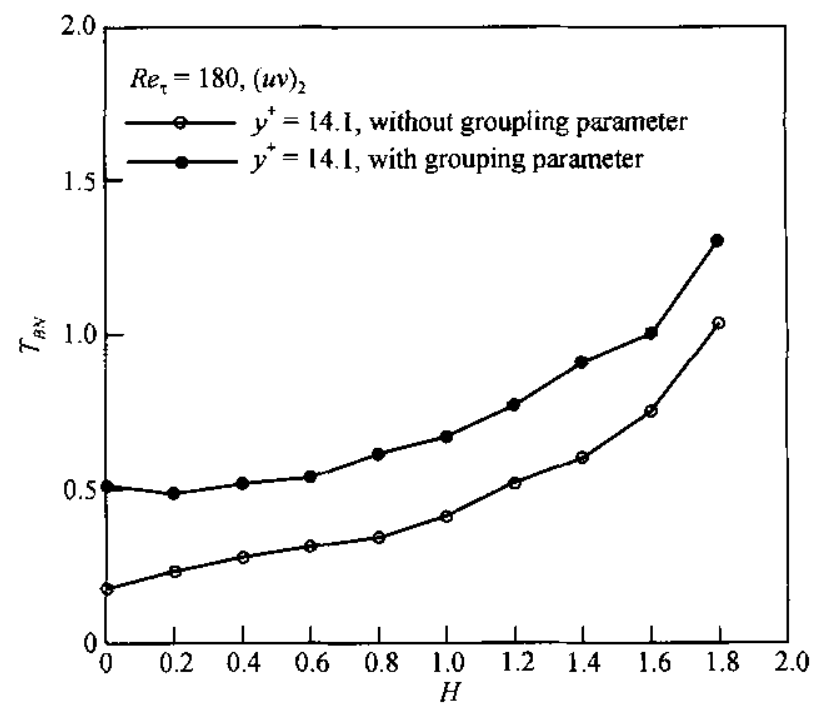

Fig. 11. Comparisons between burst periods with and without grouping parameter, $(u v)_{2}, R e_{\tau}=180$. 
Fig. 12 presents the pdf curves of time separation between ejections detected by $(u v)_{2}$ method at $\operatorname{Re}_{\tau}=180, y^{+}=14.1,19.7$, and $H=1.0$. In this figure, the positions of the grouping parameters are indicated by the arrows. Fig. 13 shows the average burst period detected by introducing the grouping parameter determined this way. It can be seen that, $T_{B N} \approx 0.5$ for $0<H<1.0$. In the similar way, the average burst period can be obtained by mu-level method, as shown in Fig. 14. For mu-level method, $T_{B N} \approx 0.5$ for $0<L<1.0$.

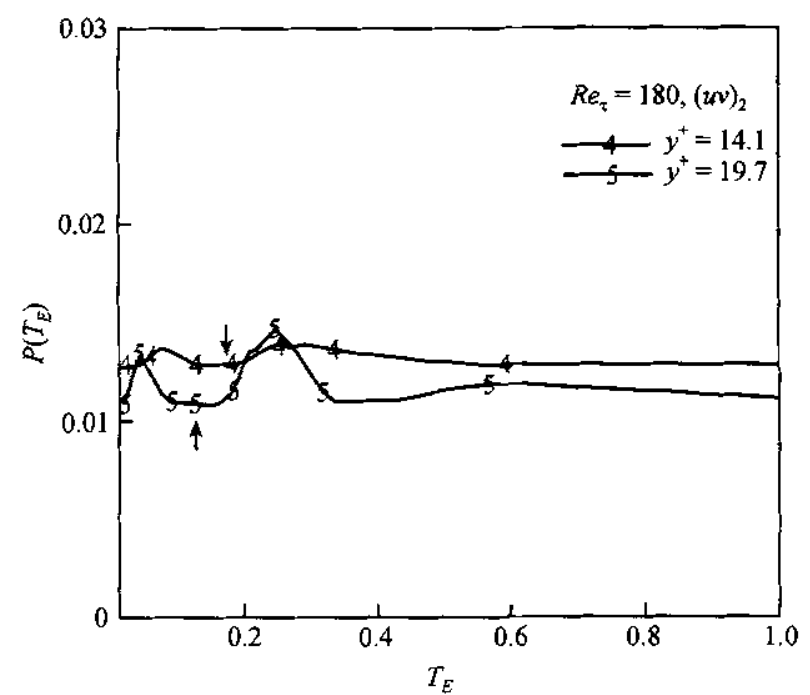

Fig. 12. Smoothed pdf curve of time separations between ejections, $(u v)_{2}, R e_{\tau}=180$.

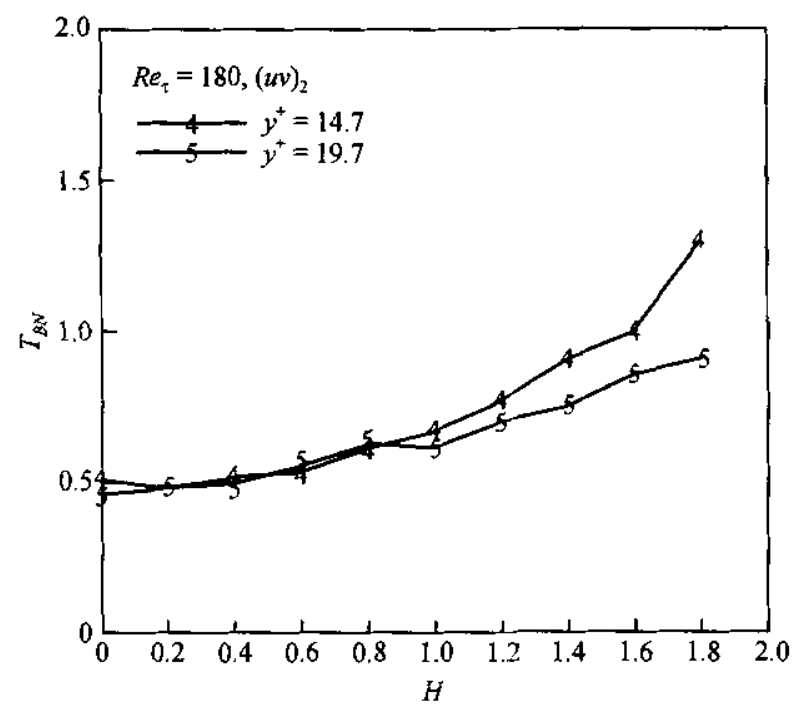

Fig. 13. Average burst periods detected by $(u v)_{2}$ method, $R e_{\tau}=180$.

In addition, we have simulated two other cases, namely, the channel flows at $R e_{\tau}=$ 300 and 395. Also, the average burst periods are detected by using the procedure mentioned above. Fig. 15 depicts the variations of non-dimensional burst period with Rey- 


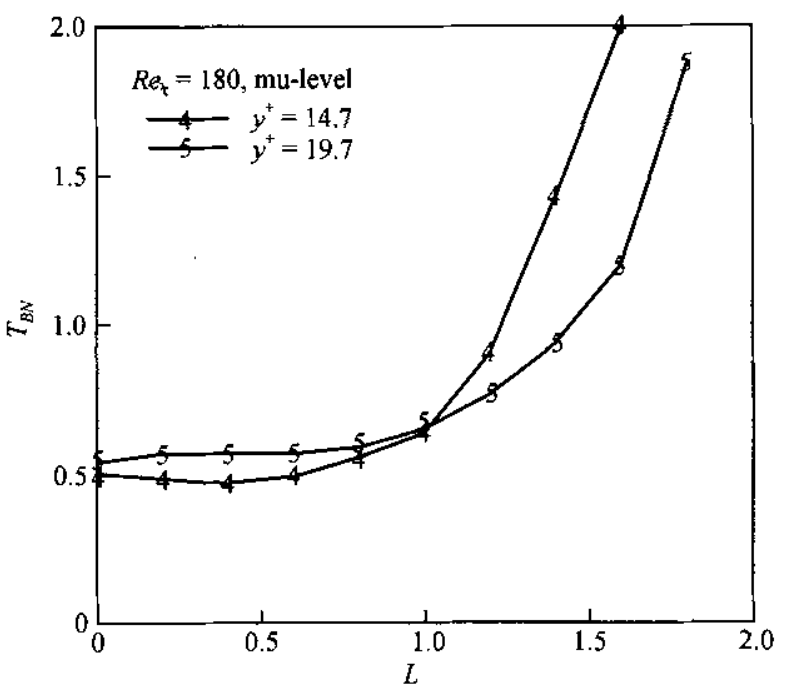

Fig. 14. Average burst periods detected by mu-level method, $R e_{\tau}=180$.

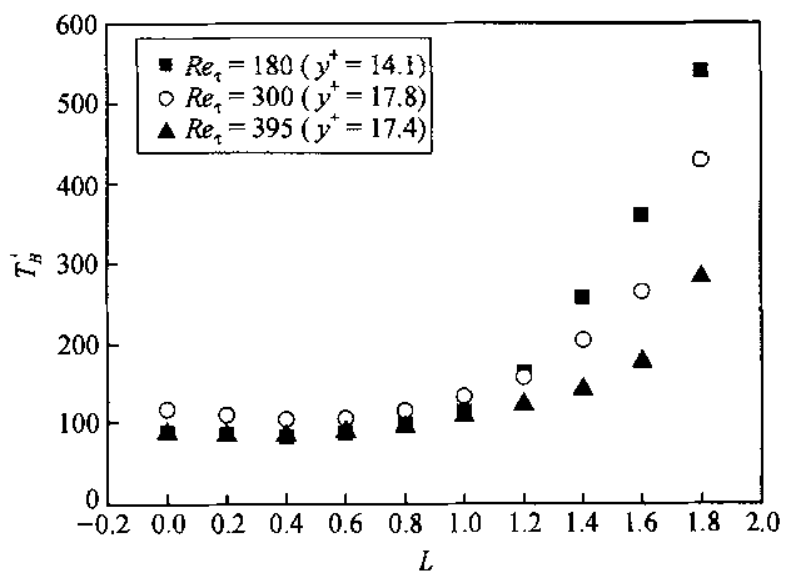

Fig. 15. Variations of non-dimensional burst period detected by mu-level method with Reynolds number.

nolds number by mu-level method. Obviously, when $0<L<1$, the non-dimensional burst period does not vary with Reynolds number, i.e. $T_{B}^{+} \approx 90$, in good agreement with the results by Luchik \& Tiederman ${ }^{[7]}$. When $L>1$, the non-dimensionalized burst periods of three Reynolds numbers start to deviate from the value of 90 . Hence, the non-dimensionalized burst period hardly exhibits dependence on Reynolds number in a certain parameter region, i.e. $0<L<1$.

\subsection{Burst-to-bed area ratio}

In the experiment of interaction between sediment particles and near-wall turbulence, Nino \& Garcia $^{[4]}$ found that the finer sediment particles accumulate around the lowspeed streaks in the flows on a smooth wall, and they are entrained to the outer flows by the lift, oscillating and breakdown of the low-speed streaks in these regions. Cao ${ }^{[17]}$ 
worked out a pick-up formula with sound physical background based on the temporal and spatial scales of burst events. However, the spatial characteristic, the burst-to-bed area ratio is fairly rough in his formula. Hence, we calculate the burst-to-bed area ratio in the near-wall region based on the LES databases by using conditional sampling.

By analyzing the turbulent signal series of all grid points in a given horizontal plane, counting all the points at which an ejection event is happening and dividing the number of such points by the total grid number in horizontal plane, one obtains the burst-to-bed area ratio $A$. Figs. 16 and 17 show the variations of burst-to-bed area ratio detected by $(u v)_{2}$ and mu-level methods with threshold at three Reynolds numbers. All detected planes locate in the buffer layer, where the bursts prevail. Generally speaking, the burst-to-bed area ratios detected by two techniques vary in a similar way with the threshold. The larger the thresholds, the more intense the fluctuations detected and the smaller the burst-to-bed area ratio. When $0<H<1$, the burst-to-bed area ratios detected by $(u v)_{2}$ method vary from $10 \%$ to $30 \%$, while when $0<L<1$, the ratios detected by

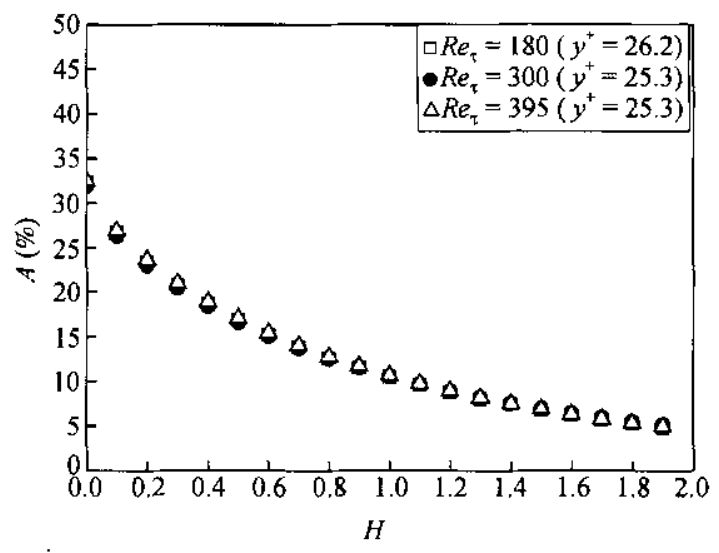

Fig. 16. Burst-to-bed area ratio detected by $(u v)_{2}$ method.

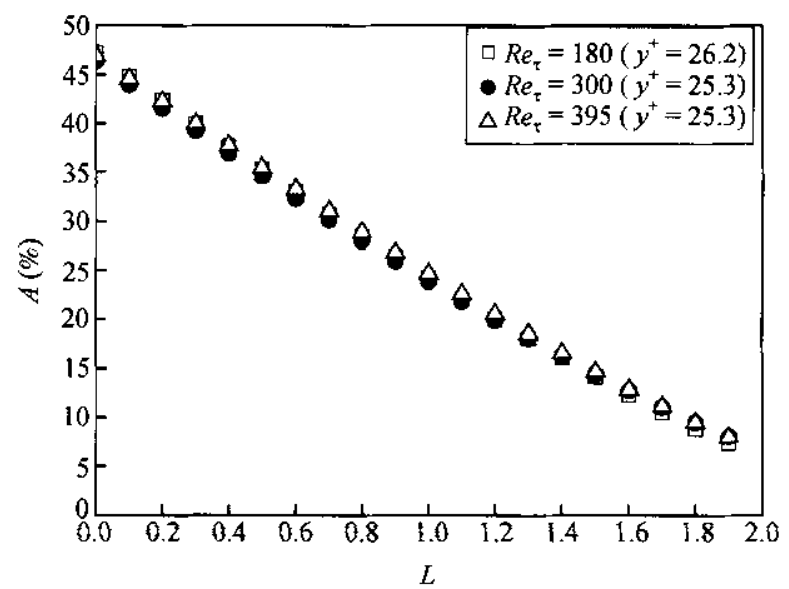

Fig. 17. Burst-to-bed area ratio detected by mu-level method. 
mu-level method vary from $25 \%$ to $45 \%$. Because two techniques detect different characteristics of the turbulent burst process, the burst-to-bed area ratios detected by them change correspondingly. The normal velocity component is taken into consideration additionally in the sampling function of $(u v)_{2}$ method. Therefore, the ejection events detected by $(u v)_{2}$ method are part of those by mu-level method. And it results in the difference between the burst-to-bed area ratios by them. In the current range of Reynolds number, no effect of Reynolds number has been found on the burst-to-bed area ratio.

\title{
3 Conclusion
}

The LES databases of turbulent channel flows have been established to investigate the burst process. The current LES databases are verified and validated by comparison with generally accepted DNS results. By analyzing the proportion of SGS stress in total stress, the validity of the current LES databases for the detection of turbulent bursts is also proved. Several LES databases at different Reynolds numbers are utilized to examine the temporal and spatial characteristics of burst process.

(1) The average burst periods detected by traditional conditional sampling methods greatly depend on the thresholds. A method to determine the grouping parameter from the pdf curve of time separations between ejections is proposed. With such grouping parameters, the average burst periods vary very little with the thresholds in quite a wide range of threshold $\left(0<H<1\right.$ in $(u v)_{2}$ method, $0<L<1$ in mu-level method).

(2) The burst-to-bed area ratios are obtained. By analyzing average burst periods and burst-to-bed area ratios at different Reynolds numbers, we have found that both of them are basically independent of Reynolds number.

\begin{abstract}
Acknowledgements This work was supported by the National Natural Science Foundation of China (Grant Nos. 10332050 and 10002023), and Innovation Project, CAS. The authors gratefully acknowledge the support of the Opening Research Foundation of the State Key Laboratory of Water Resources and Hydropower Engineering Science (Grant No. 2003B003).
\end{abstract}

\section{References}

1. Kline, S. L., Reynolds, W. C., Schraub, F. A., Runstadler, P. W., The structure of turbulent boundary layers, J. Fluid Mech., 1967, 30(4): 741—773.

2. Kim, J., Moin, P., Moser, R. D., Turbulence statistics in fully developed channel flow at low Reynolds number, J. Fluid Mech., 1987, 177: 133-166.

3. Gyr, A., Schmid, A., Turbulent flows over smooth erodible sand beds in flumes, J. Hydr. Res. IAHR, 1997, 35(4): 525-544.

4. Nino, Y., Garcia, M. H., Experiments on particle-turbulence interactions in the near-wall region of an open channel flow: implications for sediment transport, J. Fluid Mech., 1996, 326: 285-319.

5. Sun, K. H., Shu, W., On the burst detection techniques in wall-turbulence, Acta Mechanica Sinica (in Chinese), 1994, 26(4): 488-493.

6. Bogard, D. G., Tiederman, W. G., Burst detection with single-point velocity measurements, J. Fluid Mech., 1986, 162: 389-413.

7. Luchik, T. S., Tiederman, W. G., Timescale and structure of ejections and bursts in turbulent channel flows, J. Fluid Mech., 1987, 174: 529—552. 
8. Kim, J., Spalart, P. R., Scaling of the bursting frequency in turbulent boundary layers at low Reynolds numbers, Phys. Fluids, 1987, 30(11): 3326-3328.

9. Li, L., Xu, C. X., Zhang, Z. S., Study of burst phenomena in wall turbulence by wavelet analysis, Acta Mechanica Sinica (in Chinese), 2001, 33(2): 153-162.

10. Zang, T. A., Numerical simulation of the dynamics of turbulent boundary layers: Perspectives of a transition simulator, Philos. Trans. R. Soc., London, Ser. A, 1991, 336: 95-102.

11. Piomelli, U., 2001 Large-eddy and direct simulation of turbulent flows, Short course delivered at CFD2001-9e conference annuelle de la Societe canadienne de CFD, Kitchener, Ontario.

12. Jiang, N., Wang, Z. D., Shu, W., The maximum energy criterion for identifying burst events in wall turbulence using wavelet analysis, Acta Mechanica Sinica (in Chinese), 1997, 29(4): 406-411.

13. Moser, R. D., Kim, J., Mansour, N. N., Direct numerical simulation of turbulent channel flow up to $R e_{\hat{o}}=590$, Phys. Fluids, 1999, 11(4): 943-945.

14. Blackwelder, R. F., Haritonidis, J. H., Scaling of the bursting frequency in turbulent boundary layers, J. Fluid Mech., 1983, 132: 87-103.

15. Alfredsson, P. H., Johansson, A. V., Time scales in turbulent channel flow, Phys. Fluids, 1984, 27(8): 19741981.

16. Shah, D. A., Antonia, R. A., Scaling of the "bursting" period in turbulent boundary layer and duct flows, Phys. Fluids A, 1989, 1(2): 318-325.

17. Cao, Z., Turbulent bursting-based sediment entrainment function, J. Hydr. Engng. ASCE, 1997, 123: 233236. 